\title{
Diseño de Guías para Laboratorios Virtuales de Física
}

\section{Guidelines Design for Virtual Laboratories}

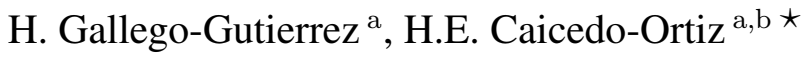 \\ ${ }^{a}$ Grupo de Ingeniería y Tecnologías Cuánticas, Facultad de Ingeniería, Corporación Universitaria Autónoma del Cauca, \\ Popayán, Colombia. \\ ${ }^{\mathrm{b}}$ Instituto de Educación Media Superior del Distrito Federal, Ciudad de México, México.
}

Recibido: 15-09-2011; revisado: 8-11-2011; aceptado: 17-1-2012.

\begin{abstract}
Resumen
Presentamos una metodología que permite la creación de los contenidos que acompañan a un grupo de diez simulaciones construidas con Easy Java Simulation y que, en conjunto, forman una guía para la elaboración de laboratorios virtuales en la enseñanza del curso de Física I.

Palabras Claves: Simulaciones, Laboratorios Virtuales, Easy Java Simulation, Física Educativa, Guías de Laboratorio.

Abstract

We present a methodology that enables the creation of content that accompany a group of ten simulations built with Easy Java Simulation and which together form a guide for the development of virtual laboratories in teaching Physics I course.
\end{abstract}

Keywords: Simulations, Virtual Labs, Physics Education, Laboratory Guides.

\section{Introducción}

En el proceso de enseñanza-aprendizaje de la Física, resulta relevante el proceso de experimentación por parte de los estudiantes, ya que este juega un papel fundamental en comprensión y asimilación de los conceptos físicos estudiados en los distintos cursos teóricos. Las prácticas experimentales en los cursos de Física desarrollan destrezas básicas en el alumno como el correcto uso y apliación de las herramientas de la Física experimental, aprender el tratamiento de datos, manejar conceptos básicos, comprender el rol de la observación directa en Física y diferenciar entre las inferencias que se llevan a cabo a partir de la teoría y las obtenidas a partir de la práctica.

El paradigma del laboratorio se ha extendido en los últimos años, por lo cual es posible que las prácticas de laboratorio puedan realizarse de forma convencional en la cual exista contacto físico y pueda manipular los elementos, dispositivos e instrumental requeridos para el experimento (laboratorio real) o empleando simulaciones interactivas (laboratorio virtual). Cualquiera de las dos metodologías hace necesario que el estudiante realice un proceso de auto preparación de su práctica con material diseñado por los docentes, ya sea en formato electrónico o a través de materiales impresos. Algunos estudios al respecto presentan que el trabajo en estos dos ambientes es complementario y mejora el proceso de asimilación y compresión de los conceptos en los estudiantes [1].

El uso de las TIC en la enseñanza no mejora por sí solo el proceso de enseñanza-aprendizaje, por lo cual es necesario hacer uso de un enfoque pedagógico adecuado que permite el correcto uso de estas herramientas en la enseñanza de la física, lo cual se ve reflejado en un correcto diseño e implementación de las prácticas de

^ hecaicedo@gmail.com 
laboratorio.

El auge en el uso de internet y su utilización como herramienta educativa en el campo de las ciencias básicas ha permitido el surguimiento en los últimos años del uso de animaciones interactivas como elementos complementarios que mejoran la calidad, así como la forma en que los estudiantes aprenden. Cuando estas animaciones ofrecen la capacidad de retroalimentación, es posible desarrollar con ellas laboratorios virtuales [2]3].

En este contexto, en este trabajo presentamos el desarrollo de una metodología para el diseño de guías de laboratorios virtuales, en las cuales se hace uso de simulaciones computacionales de sistemas físicos desarrolladas en Easy Java Simulation [4[5]. Para el desarrollo de las prácticas se consideró el contenido programático estándar del curso de Física I - Mecánica Newtoniana ofrecido en 80 programas de Ingeniería en 25 universidades colombianas. Se emplea como referencia para la elaboración de estos cuestionarios los libros de texto de Serway [6], Alonso y Finn [7], Hewitt [8] y Kleppner and Kolenkow [9].

\section{Estructuras de las Guías}

Tomando como referencia que el alumno del curso de Física I, en su proceso de aprendizaje debe emplear distintas herramientas y metodologías que le permitan tener una correcta conceptualización de los principios físicos de estudio, las guías de los laboratorios virtuales pretenden reunir de forma interactiva estos elementos y sirviendo de complemento en el proceso de aprendizaje y afianzamiento de los principios físicos elementales.

\subsection{Conceptos Teóricos}

Como primer elemento, es necesario que el alumno posea unos conocimientos previos que le permitan llevar a cabo la práctica virtual propuesta en el laboratorio. A diferencia de proyectos como el desarrollado por el Prof. Ángel Franco en la Universidad del País Vasco 2 en el presente trabajo no se desarrolla un extenso contenido teórico de la temática. Esta decisión radica en el hecho de que en la actualidad y debido a la proliferación de información por internet, los estudiantes ya no hacen uso de los libros de texto y están expuestos a adquirir información que no siempre tiene gran fiabilidad. Por este motivo, en la web del laboratorio desarrollado en este trabajo, se incluye una breve introducción a los conceptos físicos involucrados así como referencias es-

\footnotetext{
2 http://www.sc.ehu.es/sbweb/fisica/
}

pecíficas de libros de texto de amplio reconocimiento internacional, las cuales deben ser consultados por los estudiantes antes de realizar su práctica virtual, constituyendo esta etapa su pre-laboratorio. La idea desde un principio es que al igual que en la clase teórica, en la cual el estudiante está acompañado de su libro, también lo realice en las prácticas de laboratorio y en las practicas de los laboratorios virtuales, convirtiéndose esta en una herramienta fundamental para la mejor asimiliación de los conceptos físicos.

\subsection{Descripción de la Simulación}

En un segundo ítem, el estudiante encuentra una corta descripción de lo que hace la simulación. Se espera que con eso el estudiante despierte su interés e inicie un proceso de experimentación que le permite conocer más sobre qué puede y qué no puede hacer con la simulación.

\subsection{Simular}

Como tercera etapa, el estudiante se encuentra ante una serie de instrucciones que le permiten interactuar ya directamente con la simulación del fenómeno físico de interés. En las dos primeras instrucciones, se invita al estudiante a que interactué de forma libre y espontanea con la simulación, conociendo su funcionamiento, cambiando parámetros y determinando los límites y alcances que posee el applet. A continuación se describe una serie de pasos con preguntas sobre ciertos resultados que arrojan las simulaciones para casos específicos.

\subsection{Consultar}

Haciendo uso de un libro de texto en Física, del laboratorio virtual y de la WWW, el estudiante debe responder una serie de preguntas de tipo conceptual, las cuales permiten tener una mejor compresión de los conceptos desarrollados y estudiados en la práctica el laboratorio virtual.

\subsection{Solucionar}

Finalmente, se plantea a los estudiantes una serie de problemas escogidos de distintos libros de texto, que permitirán en forma conceptual y operativa tener una mejor compresión del fenómeno físico de estudio. 
A excepción del último ítem, los estudiantes deben enviar sus respuestas vía electrónica haciendo uso del sistema google-apps que posee el sistema de correo electrónico de la Corporación Universitaria Autónoma del Cauca al correo del docente.

\section{Actividades a Desarrollar en el Laboratorio Virtual}

Se presentan a continuación las guías desarrolladas para cada uno de los laboratorios virtuales creados en este trabajo de grado

\subsection{Guía de Tiro Semi-Parabólico}

\subsubsection{Descripción}

Se observa el comportamiento de un proyectil que es lanzado desde un avión con velocidad inicial 0 en el eje vertical (y) y velocidad inicial horizontal (x) igual a la del avión. Se observa la trayectoria del proyectil, la cual es semiparabólica. Se puede cambiar la altura a la cual se encuentra el avión, así como la velocidad horizontal del avión y del proyectil (Vease Figura 11).

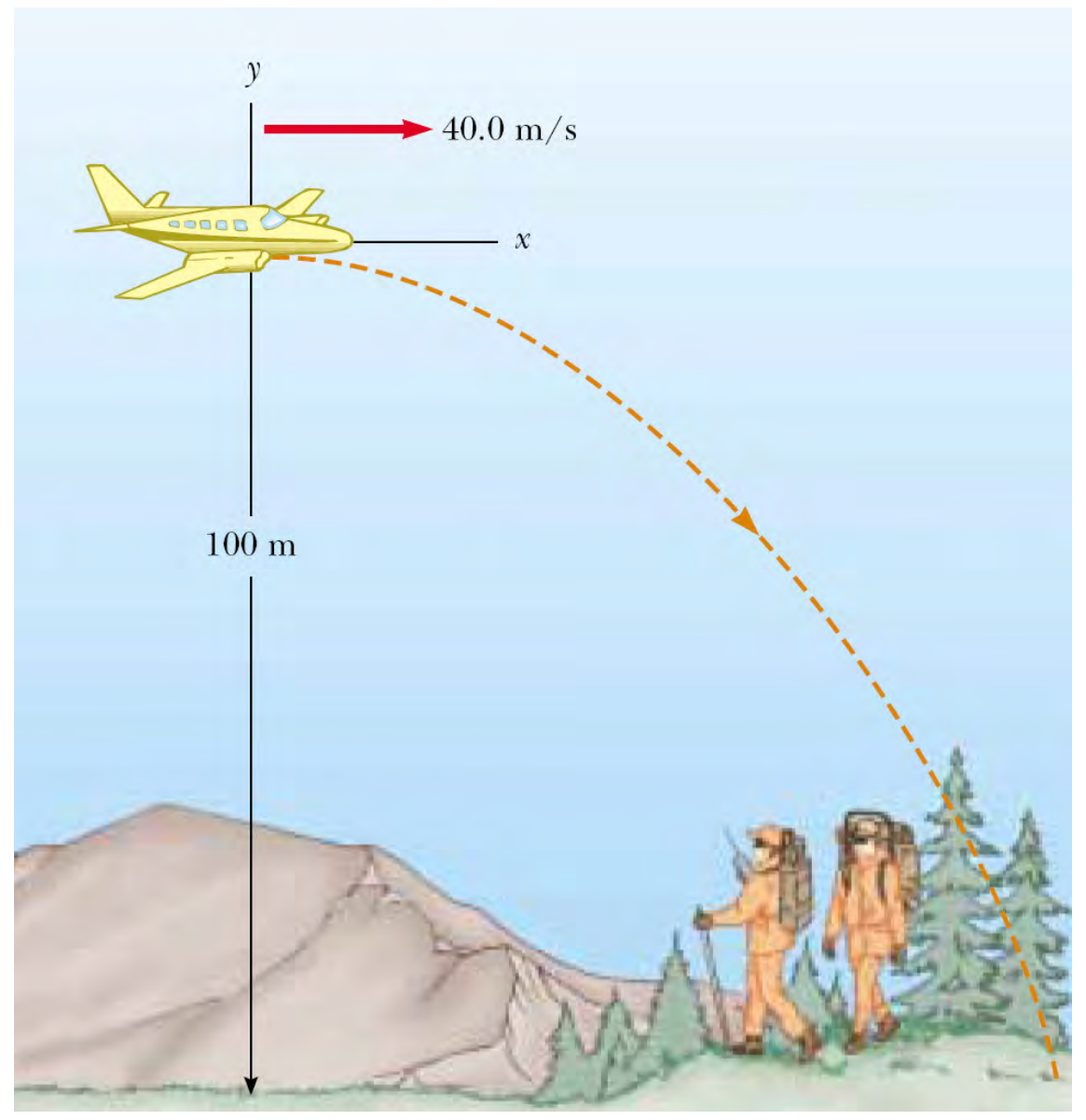

Figura 1. Representación gráfica del tiro semiparabólico. Imagen tomada de [6].

\subsubsection{Simular}

1. Empleando el mouse, juegue y familiarícese con la simulación presentada en la guía.

2. ¿Qué elementos de la simulación pueden ser cambiados por el usuario?

3. Corra la simulación para 10 valores distintos de velocidad. ¿Qué observa?

4. Corra la simulación para un cierto valor de velocidad. Detenga a mitad de camino la simulación con el botón parar. Cambie el valor de la velocidad a una menor a la inicialmente escogida y ejecute la opción simular nuevamente. ¿Qué ob- 
serva?

5. Corra la simulación para un cierto valor de velocidad. Detenga a mitad de camino la simulación con el botón parar. Cambie el valor de la velo- cidad a una menor a la inicialmente escogida y ejecute la opción simular nuevamente. ¿Qué observa?

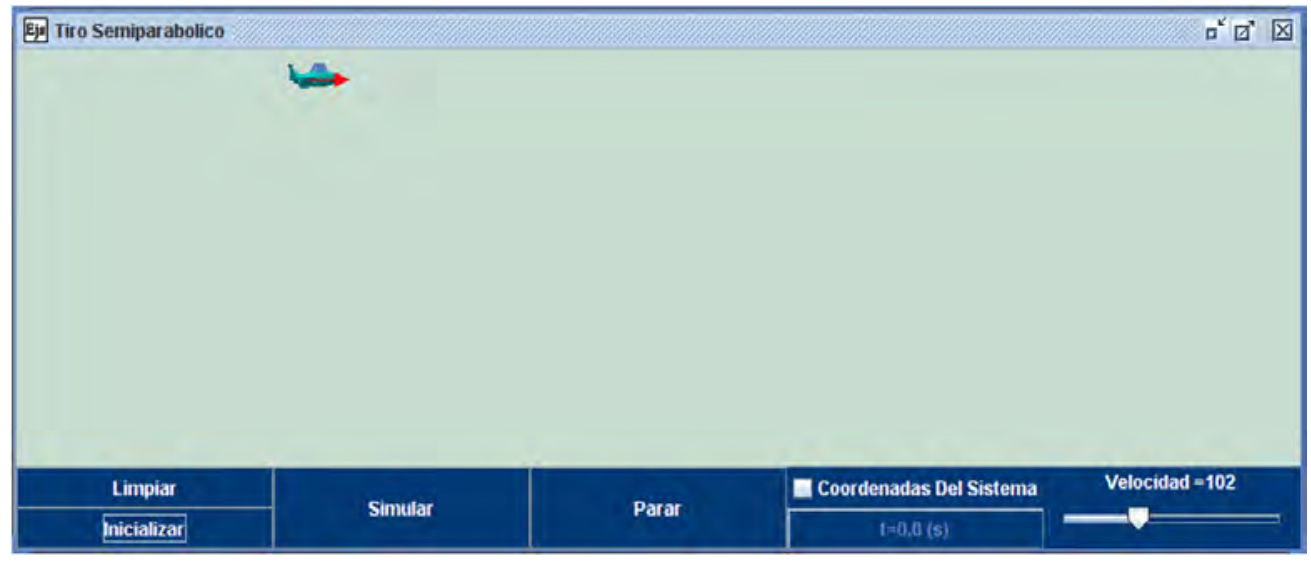

Figura 2. Simulación del tiro semiparabólico realizada en Easy Java Simulation.

\subsubsection{Consultar}

1. Se deja caer un paquete desde un avión en vuelo horizontal respecto a la superficie terrestre. Si se desprecia la resistencia del aire ¿Cómo vería el piloto el movimiento del paquete? y ¿un observador situado en tierra?

2. Con independencia de la velocidad inicial, el movimiento de un proyectil (si se desprecia la resistencia del aire) está siempre contenido en un solo plano, ¿por qué?

3. Se lanza un proyectil espacial tripulado en una trayectoria parabólica. ¿Por qué siente ingravidez un hombre dentro de la cápsula? ¿en qué sentido se dice que no pesa?

4. Se puede jugar a atrapar una pelota ligera en un avión en vuelo horizontal como si el avión estuviera en reposo. ¿Sigue siendo esto posible cuando el avión realiza un giro?

\section{Test de Evaluación de los Laboratorios Virtuales}

La prueba de la aplicación consistió en invitar a estudiantes de los programas de Ingeniería de Sistemas, Ingeniería Electrónica e Ingeniería Ambiental y Sanitaria a visitar y explorar el sitio web en el cual se incor-

\subsubsection{Solucionar}

1. Un avión deja caer una partícula con una velocidad de $46 \mathrm{~m} / \mathrm{s}$ haciendo un ángulo de $30^{\circ}$ por encima de la horizontal. Calcular el alcance, el tiempo de vuelo, y las componentes de la velocidad de la partícula. Hallar también la altura máxima.

2. Un avión deja caer una partícula desde una altura de $200 \mathrm{~m}$ con una velocidad de $46 \mathrm{~m} / \mathrm{s}$ haciendo un ángulo de $30^{\circ}$ por encima de la horizontal. Calcular el alcance, el tiempo de vuelo, y las componentes de la velocidad de la partícula al nivel del mar. Hallar también la altura máxima. (Hallar primero, las componentes horizontal y vertical de la velocidad inicial)

3. Un aeroplano que vuela a $100 \mathrm{~m} / \mathrm{s}$ deja caer una caja desde una altura de $200 \mathrm{~m}$

a) ¿Cuánto tarda la caja en llegar a la tierra?

b) ¿Qué distancia recorre horizontalmente mientras cae?

c) Calcule las componentes horizontal y vertical de su velocidad cuando llega al suelo.

poraron las 10 guías de laboratorio. Se les suministró a cada uno de ellos una encuesta con diez preguntas. Cada pregunta debía ser calificada de 1 a 5 siendo 1 la peor nota, 5 la mejor y 3 aprobatoria.

La muestra total de estudiantes considerados para el proceso de prueba de la aplicación fue de 41 personas. 


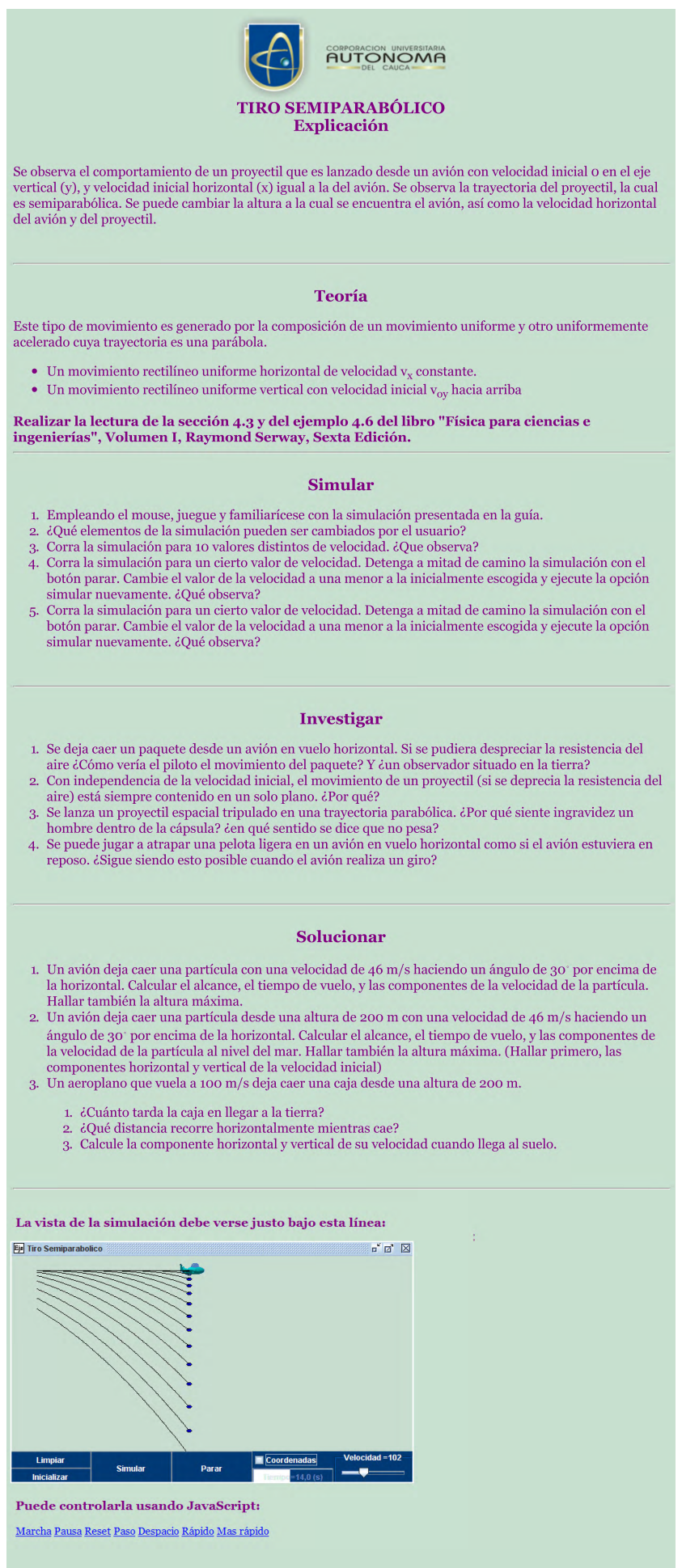

Figura 3. Guía de laboratorio virtual sobre tiro semiparabólico.

No se hace una discriminación en el sexo (Masculino o Femenino) ni en las carreras que cursan los encuestados. Así mismo, los contenidos programáticos corres- pondientes al curso de Física I son los ofrecidos en la Corporación Universitaria Autónoma del Cauca durante el año 2008. 
Los resultados obtenidos en la encuestas se resumen así:

1. Un alto procentaje de los estudiantes piden la presencia de fórmulas y valores numéricos, para comprender mejor el uso de las simulaciones.

2. Los estudiantes afirman que en la sección consultar existen preguntas que no está incluidas en los temas propuestos en el pensum académico.

3. Los encuestados piden incluir mayor interactivida, como animaciones al interior de la simulación.

4. Un gran porcentaje superior al $50 \%$ consideran los laboratorios virtuales una herramienta muy útil a la hora de recrear los fenómenos físicos.

5. Las simulaciones muestran de forma clara los efectos físicos a demostrar.

6. Unos cuantos estudiantes $(10 \%)$ les gustaría encontrar en las guías de los laboratorios virtuales mayor contenido teórico, asi como un mayor número de modelos matemáticos.

7. Un bajo porcentaje $(0,5 \%)$ de los estudiantes piden mejorar la interfaz gráfica.

8. Para trabajar con las simulaciones, varios estudiantes $(5 \%)$ afirman que es necesario tener una conexión a internet de alta velocidad.

\section{Conclusiones}

Se logra diseñar de manera eficiente, las guías que acompañan a cada una de las simulaciones, considerando para tal fin parámetros pedagógicos y recomendaciones referentes a investigaciones sobre la enseñanza de la Física.

Haciendo uso del dinamismo propuesto por el empleo de los laboratorios virtuales de Física en java en la enseñanza de esta área, el estudiante desarrolla conocimientos y habilidades que le permiten tomar decisiones frente a problemas complejos como son, las representaciones del fenómeno natural del mundo real y lo preparan para escenarios futuros en los que se va a competir como profesional.

El uso de los computadores en la enseñanza de la Física y en especial el desarrollo y uso de laboratorios virtuales, proporciona una mejora fundamental del aprendizaje conceptual de los estudiantes.
El uso de la herramienta Easy Java Simulations, ofrece un punto de partida para el desarrollo de aplicaciones de alta calidad en el campo de la Física, abriendo nuevas posibilidades en el proceso de diseño de un fenómeno, así como estrategia de aprendizaje y una forma muy práctica de hacer ciencia.

El diseño y producción de laboratorios virtuales hace posible la adquisición de nuevo conocimiento, sin la necesidad de tener los medios económicos para adquirir costosos laboratorios.

El proceso de modelado de un fenómeno físico, permite el entendimiento y la comprensión de los temas que sustentan su desarrollo. Adicionalmente, también admite tener un análisis con detalle del comportamiento del fenómeno simulado y por último obtener una interfaz con creatividad propia.

La combinación de un entorno interactivo dotado de animaciones en el desarrollo de laboratorios virtuales para la enseñanza de la Física promueve la participación activa de los estudiantes en su propio proceso de aprendizaje.

\section{Referencias}

[1] Lucero et al I. Trabajo de laboratorio de Física en ambiente real y virtual. Memorias Comunicaciones Científicas y Tecnológicas, UNNE, Argentina, 2000.

[2] Konaté, Dialla. Mathematical Modeling, Simulation, Visualization and e-Learning. Springer - Verlag, Berlin, $1^{\text {st }}$ edition, 2008

[3] Laboratorios Virtuales de Microsoft TechNet. http://www.microsoft.com/latam/technet/virtuallab/default.mspx, Consultado el 2 de Mayo de 2011.

[4] Francisco Esquembre. Creación de simulaciones interactivas en Java. Pearson Educación, España, 1era edición, 2005.

[5] Francisco Esquembre. Easy java simulations: a software tool to create scientific simulations in java. Computer Physics Communications 156, 156:199-204, 2004.

[6] Raymond Serway et al. Física para ciencia e ingenierías Volumen I. Thomson, Mexico, Sexta edición, 2005.

[7] Alonso, M. and Finn, E. Fisica I. Addison-Wesley Iberoamericana, Mexico, 1995.

[8] Paul G. Hewitt. Fisica Conceptual. Addison-Wesley Iberoamericana, Mexico, Cuarta edición, 1995.

[9] Kleppner, Daniel and Kolenkow, Robert. An introduction to mechanics. McGraw-Hill International Editions, Engineering Mechanics Series, $1^{\text {st }}$ edition, 1987. 